\title{
An Application of Knowledge Management System for Tourism Regions
}

\author{
Yijin Shi1, a *, Zhiyang Jia1,b and Wei Gao2,c \\ ${ }^{1}$ Tourism and Culture College, Yunnan University, Lijiang, China \\ ${ }^{2}$ School of Information Science, Yunnan Normal University, Kunming, China \\ a349497232@qq.com, bjdyjazz@gmail.com, 'gaowei@ynnu.edu.cn
}

\section{Keywords: Tourism Regions; Knowledge Management System; Knowledge Entity}

\begin{abstract}
An application of knowledge management system for tourism management is presented. The knowledge system can be featured as an online web application which is capable of stores and retrieves knowledge, improves collaboration, locates knowledge sources, mines repositories for hidden knowledge, captures and uses knowledge. The knowledge management system is viewed as an effective solution to get out of the dilemma of information over-load for tourists. The system is comprised of the following parts: the personal knowledge terminals for the individuals, the PC workstation for tourism companies, the PC workstation for the managers of tourism regions, the knowledge base, the file base, a Web user interface, a WAP user interface and a mobile APP.
\end{abstract}

\section{Introduction}

Tourism regions refer to the permanent region units [1] controlled by the administration of tourism regions within a certain geographical boundary, in providing the tourism service facilities and the corresponding tourism services so that the visitors and tourists are able to enjoy the sightseeing, entertainments, culture immersion experiences and other activities. Driven by the wave of knowledge economy wave and information, the management strategy of tourism regions must be shifted as soon as possible. In this way, it is possible to adapt to the internal and external competitions in an increasingly intense way.

Knowledge refers to the relevant information in the production and management process while the knowledge of tourism regions is featured as dispersion, uncertainty and dynamics, etc. Many tourism enterprises and tourism practitioners have not been aware of the significance of the knowledge of tourism regions [2]. At present, some tourism regions go through the relatively low management level as well as the low quality and the great turnover of the practitioners. As a consequence, much knowledge is mastered by the different enterprises and individuals so that it is difficult to fully share and utilize the information. The tacit knowledge is highly personalized and difficult to format including the subjective judgment, intuitions and hunches, for example, the experience of the tourism practitioners. Explicit knowledge can be displayed in words and numbers, easily exchanged and shared in the form of data, such as the employee handbook of tourism regions edited and published by the administration of tourism regions, etc.

With the rise of the tourism industry in recent years, much tacit and explicit knowledge of tourism regions that are featured as dispersions and others cannot be effectively and timely transmitted to the tourists in causing the difficulties for the marketing of tourism regions. The tourists often go into a hobble "lots of stories, legends and versions with the rare knowledge". Based on the mass information of tourism regions, it is in urgent need to support the digital platform in the operation and management of tourism regions. Thus the knowledge management system is an effective solution to get out of the dilemma. 


\section{System Design}

Knowledge management system is an information system that can collect process and share all the knowledge of an organization with the relative technical supports including the database technology, artificial intelligence technology and computer network [3]. The system development and design is aimed at establishing the knowledge management system for tourism regions so as to solve the realistic problems such as the mass information, the dispersed knowledge, the outdated storage way, the low utilization, etc. The system can regulate the structured knowledge so that the administration of tourism regions, tourism enterprise and tourism practitioners are able to create, maintain and share the relative information of tourism regions.

System infrastructure. As shown in Fig. 1, the knowledge management system of tourism regions is consisted of the knowledge terminals for the individuals, the PC workstation for tourism enterprises, and the PC workstation for the administration of tourism regions and the servers for the storage of the knowledgebase. Its characteristics are: the personal knowledge terminals for the individuals, the PC workstation for tourism companies and the PC workstation for the managers of tourism regions are accessible to the communication network through the network connection devices and then connect to the Internet through the mobile network. In this way, it is able to build up the links with the connected knowledge base servers in implementing the query and management functions.
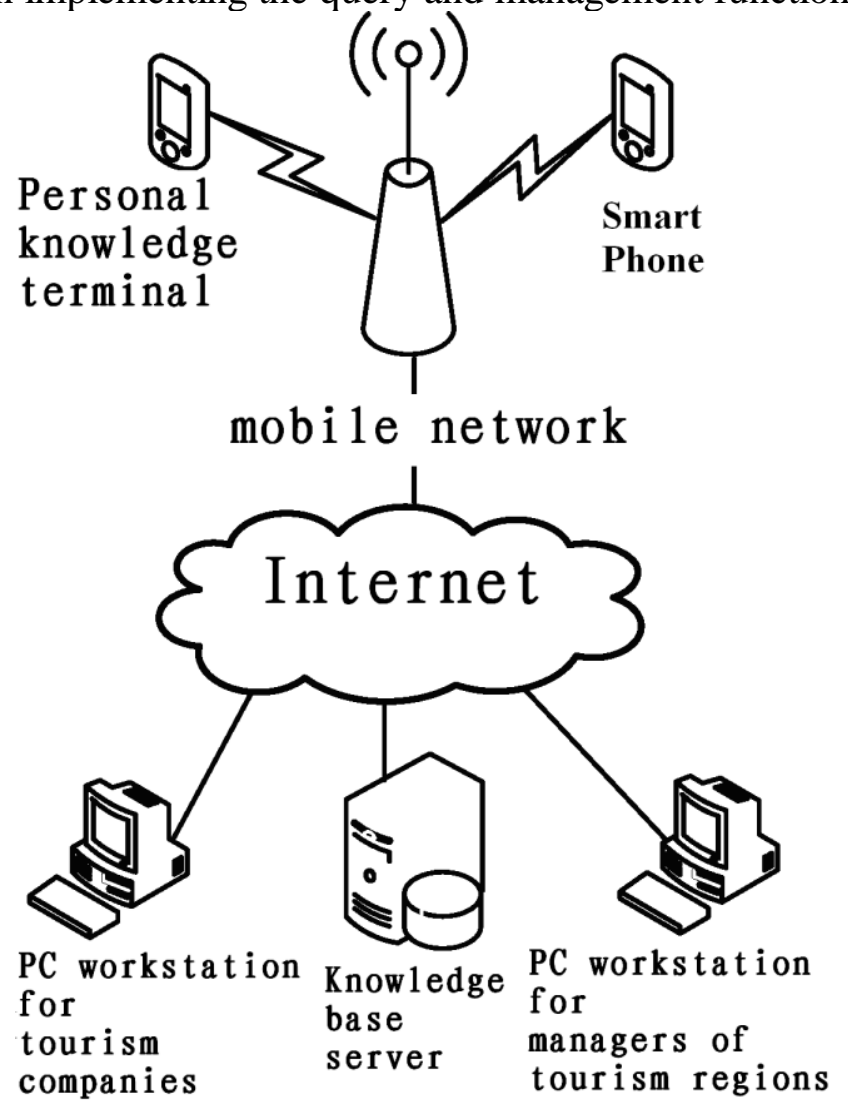

Figure 1. Finite element calculation model of arch dam and bedrock

The knowledge base server [4] is central to the knowledge management system of tourism regions. As shown in Fig. 2, the knowledge base server is consisted of the knowledge base, document library, knowledge map module, knowledge retrieval module, knowledge browse module, knowledge update module, knowledge maintenance module, file download module, file management module, WAP user interface module and Web user interface module.

Knowledge base is used to store the structured knowledge information, that is to say, to store the knowledge entity in the XML format [5]. The knowledge entity is a way of knowledge representation and storage of which the knowledgebase will store the entity title and labels, the interpretations of various versions, attachment files, comments, inter-links, etc.

The document library is to store the attachment files of all kinds of knowledge entities in the format of sound, video, image, document, etc. Knowledge map module implements the classified query 
functions on the entities through the multi-level classification and label index. Knowledge retrieval module implements the fuzzy query on the entities through the keywords. Knowledge browse module is to view the specific entity information. Knowledge update module is to add, modify and comment the entities. Knowledge maintenance module is to manage the classified information, regulate the labels, maintain the entities, manage the system users, etc. The file download module is accessible to download the attachment files of the entities. The file management module is to carry out the upload, download, deletion and other operations on the attachment files of the entities.

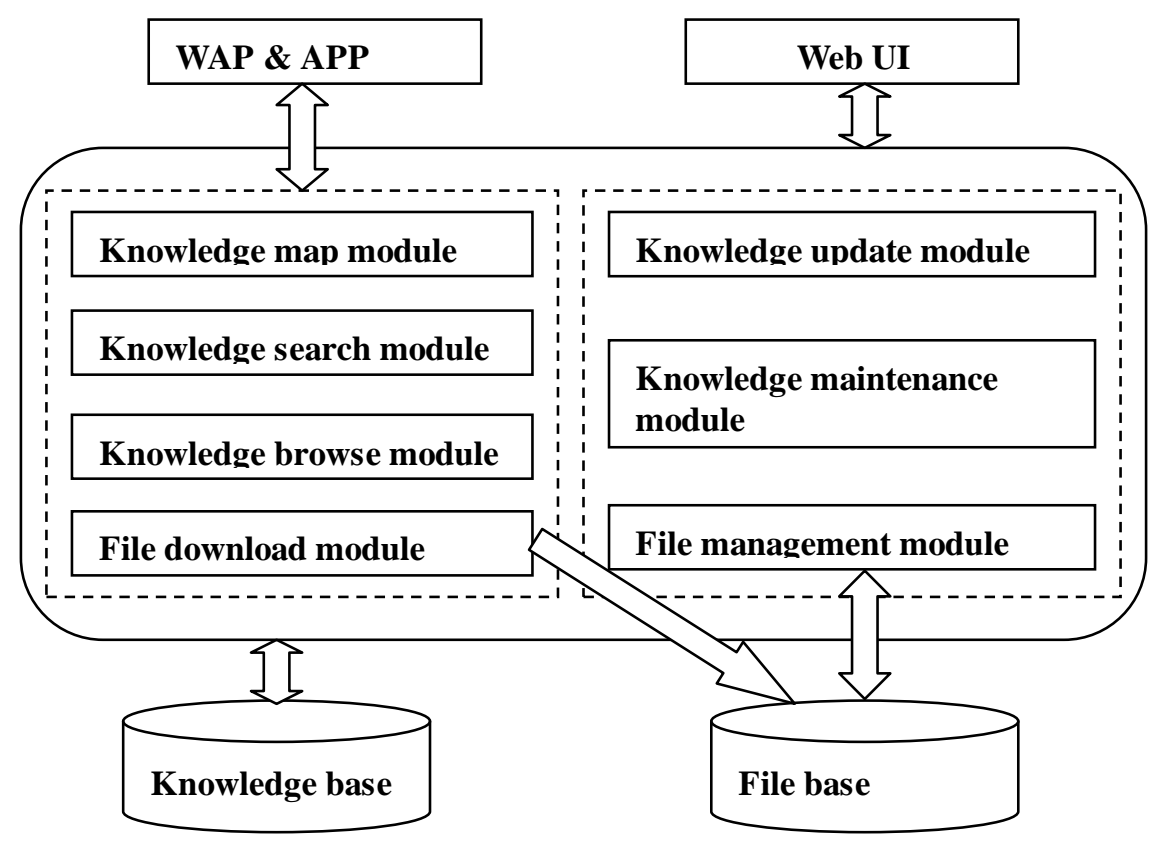

Figure 2. System infrastructure

The WAP\&APP user interface module is the interface between the individual terminals and the servers so that the individual terminals that can connect to the server are available to enter into the graphical interface based on WAP and mobile APP. The WAP\&APP user interface module is accessible to the knowledge map module, knowledge retrieval module and file download module.

The Web user interface module is to provide the graphical interface based on Web in terms of the connection of the knowledgebase servers, the PC workstation for tourism enterprises and the PC workstation for the administration of tourism regions. It is accessible to knowledge map module, knowledge retrieval module, file download module, knowledge update module, knowledge management module and file management module.

The tourism knowledgebase module at the server side supports a variety of programming design languages. Based on the optimization scheme, the WAP interface module and Web interface module adopt the ASP.NET programming language while the other modules use C\# programming language. In view of the system generality, the running and testing environments for the knowledgebase servers of tourism regions are IIS7.0 or above and Microsoft.NET Framework 3.0 version or above.

Knowledge Base and Knowledge Entity. The system architecture is shown in Fig. 1 and Fig. 2. The knowledgebase can store all the entities. Each user is able to update or comment the knowledge entities. Upon the update, the new contents will be stored in the knowledgebase as an updated version of the entity. The published user comment will be stored in the knowledgebase so that the administrator can manage the entities and comments.

In the knowledgebase, the stored entity information includes: the entity title, the interpretations for all the versions, the author and the submission date, creation time, the last update time, all the aliases, the relative text labels, all the comments, the relative attachment file lists, the category information, creation time, the inter-links, etc. The inter-links refer to the other knowledge entity list that is associated with the entity. We can take the knowledge entity "The old town of Lijiang" as an example of which its corresponding XML format is stated as follows.

$<$ entity id $=14><!-k n o w l e d g e$ entity number--> 


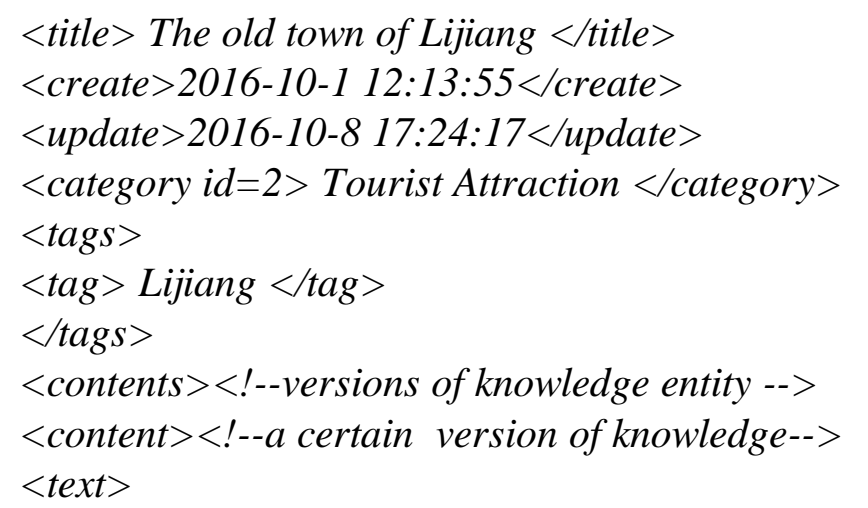

The Old Town of Lijiang is also known as Dayan Town in Lijiangba. It is an intact ancient city inhabited mostly by Naxi ethnic people and covers an area of 1.5 square kilometers. The Old Town of Lijiang was included in the UNESCO world heritage list in 1997.

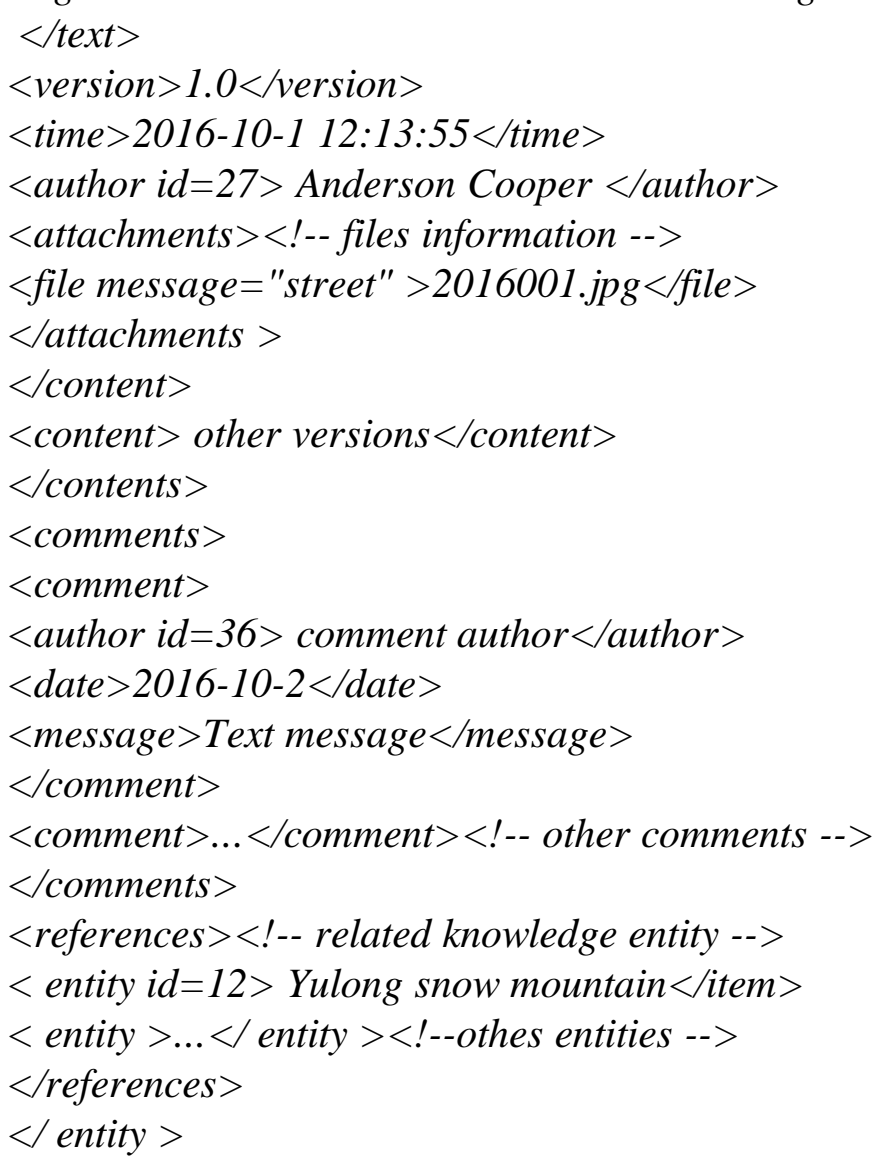

\section{Implementation of Clients}

Embedded Terminal Device. As shown in Fig. 3, the tourism knowledge terminal for the individuals is based on ARM [6] mobile devices, including the enclosures, touch screen and input keyboard. The internal components of the terminal contain CPU, SDRAM, FLASH, SD memory card, GPRS module, DM9000 network card and USB module. CPU uses S3C2440 processor; GPRS module is connected to CPU via the UART interface; DM9000 network card is used to connect the Ethernet; the USB module can be used to connect the other USB devices. 


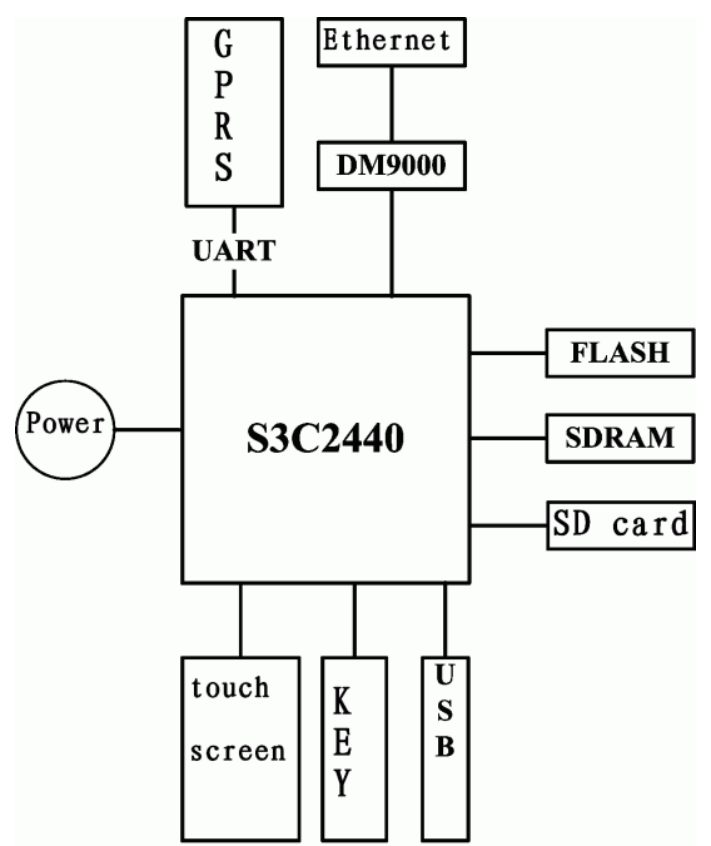

Figure 3. $\quad$ Structure of personal terminal

The personal terminal equipment of tourism knowledge uses the Windows CE operating system and installs the WAP web browser software. It is served as the WAP interface module in accessing to the knowledgebase server. The terminal is connected to the mobile communication network via the internal GPRS [7] module, and then connected to the Internet via the mobile communication network.

The PC workstations for tourism enterprises and the PC workstation for the administration of tourism regions use the ordinary $\mathrm{PC}$ with the installation of the web browser software such as Internet Explorer. It is served as the Web interface module in accessing to the knowledgebase server of tourism regions.

The mobile terminal of tourism knowledge is installed with the WAP [8] browser software. The tourism practitioners like tour guides can connect to the knowledgebase server through the individual terminals, access to the WAP interface module of the knowledgebase server of tourism regions, log in to the WAP website of the knowledgebase server, make the classification browsing based on the multi-level classifications or labels in the knowledge map module, make the entity search based on the keywords in the knowledge retrieval module, view the specific information of the entity in the knowledge browsing module, download the attachment files of the entities in the file download module, play the downloaded multi-media file, etc.

Through the connection of the PC workstations for tourism companies to the servers of tourism knowledge, the related businesses of tourism regions like the travel agency can access to the Web interface module in the server, and log in to the knowledgebase server, implement the addition, updates, comments and other operations on the entities in the knowledge update module of the server, store the knowledge into the database, upload, download and delete the attachment files of the entities in the file management module of the servers.

Through the connection of the PC workstations for the administration of tourism regions to the servers of tourism knowledge, the administration of tourism regions can enter into the knowledgebase server of tourism regions, implement the addition, updates, comments or other operations on the entities in the knowledge update module of the server, and carry out the additions, updates, comments and other operations on the entities in the knowledge maintenance module of the knowledgebase server.

Mobile app. In recent years, smart phones have evolved from unsophisticated communication devices to powerful hand-held processors. Currently, they support mastery and recall a wide range of information that encases our work and social interactions. And smart phones are reported to be used at a higher percentage for accessing and using content than for calls. Knowledge in the mobile environment is created by transforming the traditional knowledge value chain into knowledge ecology. Mobile app holds the following feathers when it developed for knowledge management [9]. 
Efficiency. Mobile applications usually require less intervention and supervision. This automation increases the efficiency of the organization promoting mobile learning.

Ubiquity. The mobility of anywhere, anytime solution 'makes mobile applications a ubiquitous platform.

Flexibility. The portability and adaptability of the mobile devices presents them as more flexible solutions.

Connectivity. The transmission and reception of data wirelessly enhances the connectivity options of the mobile devices.

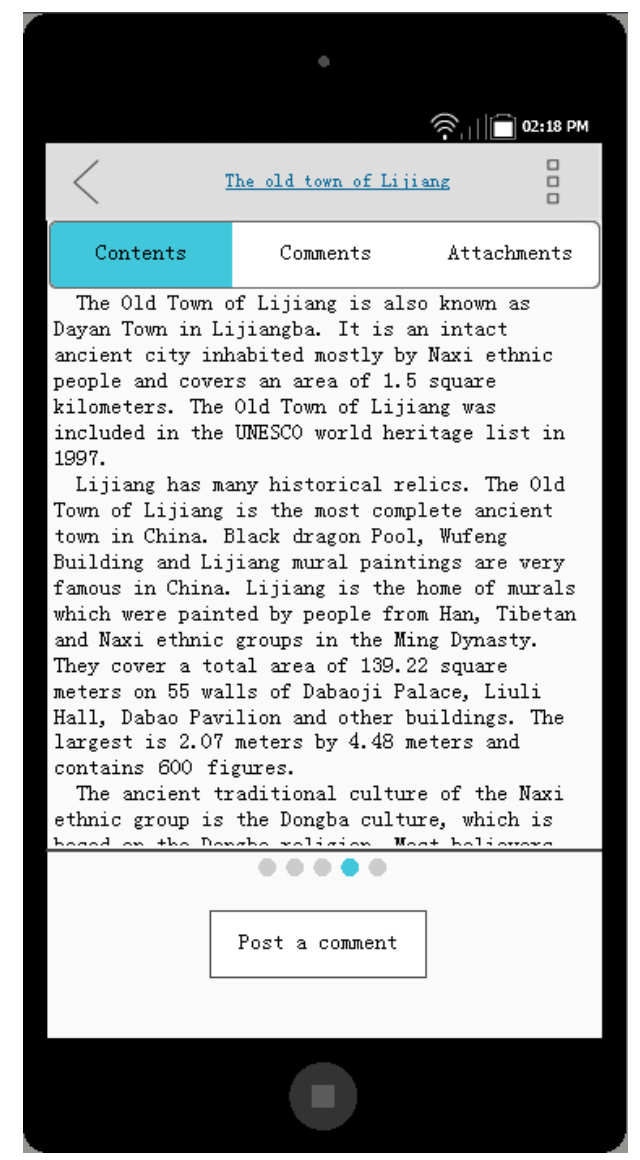

Figure 4. User interface of the mobile APP

A key feature of mobile devices is their application hosting capabilities. These applications use the device's resources to provide an array of (entertainment, utility, business, etc.) functionalities. These applications are mainly dependent on the operating system of the device. The majority of the market share of operating system is divided between Apple's iPhone OS, Android and others [10]. The client app of the knowledge management system is developed based on Android. Both the embedded terminal device and smart phone running Android can use the app to support mobile knowledge management. The user interface of the app is demonstrated in Fig. 4.

\section{Summary}

The knowledge management system of tourism regions can integrate the massive and disorganized tourism region information into the systematic and structured information. In this system, the information will be shared and utilized, improving the information transmission efficiency of tourism regions, decreasing the maintenance cost of data, improving the information storage ways of tourism regions, providing the new mode for the employee training of tourism enterprises, enhancing the professional skills of tourism practitioners, and promoting the tourist's experience of tourism regions in an effective way. 


\section{References}

[1] Stephen L.J. Smith, Regional analysis of tourism resources, Annals of Tourism Research, vol. 14, iss. 2, pp. 254-273, 1987.

[2] Zhiyang Jia, Yiyin Shi, Yuan Jia,Ding Li, A Framework of Knowledge Management Systems for Tourism Crisis Management, Procedia Engineering, vol. 29, pp. 138-143, 2012.

[3] Money, W., Turner, A. Application of the technology acceptance model to a knowledge management system, Proc. 37th Annual Hawaii International Conference on System Sciences (HICSS 04), IEEE Press, Jan. 2004, doi: 10.1109/HICSS.2004.1265573.

[4] Dehne, F., Ferreira, A.G., Rau-Chaplin, A, A massively parallel knowledge-base server using a hypercube multiprocessor. Proc. 2nd International IEEE Conference on Tools for Artificial Intelligence, IEEE Press, Nov. 1990, pp. 660 - 666, doi: 10.1109/TAI.1990.130417.

[5] Mark Craven, Dan DiPasquo, Dayne Freitag, and et al, Learning to construct knowledge bases from the World Wide Web, Artificial Intelligence, vol 118, pp: 69-113, April 2000.

[6] Segars, S, The ARM9 family-high performance microprocessors for embedded applications, Proc. International Conference on Computer Design: VLSI in Computers and Processors, IEEE Press, Oct. 1998, pp. 230 - 235, doi: 10.1109/ICCD.1998.727055.

[7] P. Lavanya, Y. Chalapathi Rao, V. Ravindra Naik, Wireless Data Acquisition System Based on ARM, International Journal of Innovative Research and Development, vol. 1, pp.14-25, August 2012.

[8] Jean-Eric Pelet, Lorna Uden, Mobile Learning Platforms to Assist Individual Knowledge Management, Lecture Notes in Business Information Processing, vol185, pp. 267-278, August 2014.

[9] Derek Flood, Rachel Harrison, Claudia Iaco, David Duce, Evaluating Mobile Applications: A Spreadsheet Case Study, Mobile Human Computer Interaction, vol. 4, iss. 4, pp. 37-65,2012.

[10] Catherine Watson, Jeff McCarthy, Jennifer Rowley, Consumer attitudes towards mobile marketing in the smart phone era, International Journal of Information Management, vol. 33, pp. 840-849, October 2013. 